\section{Recurrent pelvic organ prolapse: a case report}

Kristina Drusany Starič, Petra Bukovec, Andrej Zore, Adolf Lukanović

Division of Gynecology and Obstetrics, University Medical Centre, Ljubljana, Slovenia

\begin{abstract}
Pelvic organ prolapse (POP) is a common female condition, which is seen as protrusion of pelvic organs into or beyond the vagina. Its incidence is increasing and every woman has an $11 \%$ lifetime risk of needing surgical treatment because of this condition. POP that is recurrent several times is a rare condition; however it can have major negative impact on quality of life. We present the case of a 77-year old Caucasian woman with repeated POP. She underwent six gynecologic operations for correction of recurrent POP. Presentation of this case clearly demonstrates dramatic recurrences of POP and how difficult its treatment and prevention can be. We do not know what the reason for multiple reoccurrence of this condition might be. We need to emphasize that there are no researches on specific risk for an individual patient, which may potentiate occurrence of POP and may affect decisions about surgical procedure.
\end{abstract}

\section{Introduction}

Pelvic organ prolapse (POP) is a common gynecologic phenomenon, in which there is a protrusion of organs into or beyond the vagina due to dysfunction of suspensory ligaments and/or fascial support. ${ }^{1}$ Women with vaginal prolapse experience a dragging feeling in the vagina, back pain and protrusion from the vagina, which predispose vaginal mucosa to ulcer formation. ${ }^{2}$

There are numerous risk factors for the occurrence of POP. Bump and Norton described a model for the onset of pelvic floor dysfunction, which consists of predisposing, inciting and promoting factors. Race, anatomy and collagen distribution are considered predisposing factors. Radical surgery and vaginal delivery are due to possible damage of neuromusculature and connective tissue, which both lead to pelvic floor weakening, considered inciting factors, obesity and aging are promoting factors. ${ }^{3,4}$ Gyhagen and his colleagues found out that for each increased unit of body mass index (BMI), there was a 3\% increase in the likelihood of developing POP. ${ }^{5}$ The relative importance of other factors is yet to be determined. ${ }^{4}$ There are only a few studies in the literature that analyze factors which potentially influence prolapse recurrence. Whiteside et al. showed that women younger than 60 years and those with higher grades of prolapse have a higher risk of anatomical recurrence. However, they did not investigate the relationship with functional recurrence. ${ }^{6}$ Anatomical recurrence was also associated with complicated delivery and the presence of urinary incontinence before surgery. ${ }^{7}$

There are several treatment options for POP. ${ }^{8}$ Conservative methods were mostly found to improve symptoms of mild to moderate POP. ${ }^{9}$ For patients with a severe degree of POP and for patients to whom conservative methods of treatment did not help, surgical treatment options can be used. ${ }^{1}$ Surgery is the definitive treatment of POP. It can be reconstructive in terms of restoration of vaginal anatomy and coital function or obliterative with vaginal closure.

In case of anterior vaginal wall prolapse anterior and posterior colporrhaphy are possible. In site-specific defect repair identification and repair of specific sites of rectovaginal fascial deficiency is possible. For apical support, there are numerous possible types of operations: sacrocolpopexy, sacrocolpoperineopexy, levator myorrhaphy with apical plication, iliococcygeus fascia fixation, sacrospinous ligament suspension procedures and uterosacral ligament suspension. ${ }^{10}$ For a successful operation, it is essential to identify patients with a higher risk of recurrence after surgery to indicate individualized treatment. However, very little is known about factors associated with surgical failure. It has been reported that the higher rate of recurrence appears in the anterior compartment. ${ }^{4,11}$ Potential factors for recurrence are factors that lead to POP in the first place, preoperative grade of prolapse, type of surgery, experience of surgeons and complications in the early postoperative period. ${ }^{4}$ Despite the large number of possible surgical procedures, it is crucial that the most appropriate method is selected for each individual patient. A wrong selection of method can lead to a higher probability of re-prolapse and consequent need for reoperation.

\section{Case Report}

A 77-year old red-haired, Caucasian woman had six gynecologic surgeries due to POP. Her obstetric history includes four
Correspondence: Kristina Drusany Starič, Division of Gynecology and Obstetrics, University Medical Centre, Šlajmerjeva 3, Ljubljana, Slovenia.

Tel.: +386.68.615.579.

E-mail: drusany@yahoo.com

Key words: Recurrent pelvic organ prolapse; anterior and posterior corporaphy; sacrocolpopexy; colposuspension; total colpocleisis.

Acknowledgements: the authors would like to thank Mr. Andrew Jackson for proofreading the article.

Contributions: KDS, manuscript writing, case report selecting; $\mathrm{PB}$, manuscript writing; AZ, manuscript writing, taking photographs; $\mathrm{AL}$, manuscript writing and discussing. All authors read and approved the manuscript.

Conflict of interest: the authors declare no potential conflict of interest.

Received for publication: 2 January 2017.

Revision received: 2 May 2017.

Accepted for publication: 1 June 2017.

This work is licensed under a Creative Commons Attribution NonCommercial 4.0 License (CC BY-NC 4.0).

(C) Copyright K. Drusany Starič et al., 2017 Licensee PAGEPress, Italy

Urogynaecologia 2017; 30:194

doi:10.4081/uij.2017.194

vaginal childbirths without any complications. She had no miscarriages, blood transfusions or allergies. She had myocardial infarction in 2003. In 2005, she underwent cholecystectomy. She has been treated for arterial hypertension and diabetes type 2 (on insulin therapy) for 10 years. Her body mass index is 26 and has not changed drastically in the past few years. At the age of 50 (in 1989), she underwent her first gynecologic surgery because of primary uterine prolapse, with dragging sensation, complaint of bowel symptoms and urinary incontinence. Abdominal hysterectomy with bilateral adnexectomy and Burch colposuspension was performed. Burch colposuspension is the procedure for the treatment of stress urinary incontinence. By itself, it is not indicated for the treatment of POP, for which we use alternative treatment, in this case a hysterectomy. As we preformed the abdominal hysterectomy, we decided to preform also Burch colposuspension for further prevention of urinary incontinence. In 2006, vaginoplasty of enterocele and Richter's sacrospinous ligament fixation of vaginal vault prolapse was made, 
due to prolapse of the vaginal vault. In 2010 she again presented with total prolapse of the vaginal vault, and after the usual preoperative preparation total colpocleisis was performed. After two years due to recurrent prolapse of the vaginal vault and enterocoele, the total colpocleisis was again performed. Each time the total colpocleisis was preformed according to standards of this procedure. Results in the early postoperative period, as well as on check-up visits two months after the surgery, were perfect and she had no specific symptoms. Reoccurrence of vaginal prolapse was due to elongation of the vagina from the sides, and it happened again in 2013. Vaginal prolapse could not be satisfactorily reduced and it caused serious difficulties in passing urine and feces. The patient claimed that these problems were far worse than symptoms before previous operations. After the prolapse reduction, the vagina fell out immediately. At the end of 2013, she underwent laparoscopic sacrocolpopexy with resorbable sutures to the vagina and resorbable screws to the sacrum. On followup in February 2014, her gynecologic state was uneventful. In January 2016 recurrent vaginal prolapse was found. In June 2016 she was admitted to the Department for Gynecology for operative treatment for stage 3 POP (Figure 1) and large postoperative umbilical hernia. At that time she presented with difficult urination and defecation. The mesh could be seen eroding the vagina (Figure 2). POP Q on the examination was Aa -2.0, Ba -2.0, C -4.5, gh 3.0, tvl 8.0, Ap 3.0, Bp 3.0 (Figure 3) Perineal, vaginal and endoanal ultrasound showed loose mesh on the rectum and on the anterior wall of the vaginal vault; damaged puborectalis muscle on both sides was shown. After the usual preoperative preparation, hernia plasticity was performed. The posterior mesh was removed from the abdominal cavity. Afterwards vaginal prolapse was again corrected by total colpocleisis.

All stated surgeries took place without any complications or greater blood loss. Each time postoperative period was carried out without complications. During hospitalization, she received analgesics, antibiotics, anti-thrombotic protection, laxatives and her regular therapy. We discouraged greater physical exertion and heavy lifting for at least four months after surgery. We advised her to drink sufficient amount of fluids and to take care of regular bowel movements. Two months after surgery she had a followup visit, where she had no specific problems. All surgeries were performed by a very experienced senior surgeon.

\section{Discussion}

Pelvic organ prolapse is a common female health problem. Experts estimate that every woman has an $11 \%$ lifetime risk of needing surgical treatment because of POP. The incidence of pelvic organ prolapse is expected to increase by $46 \%$ from 2010 to year 2050. The incidence of recurrent POP is still unknown. ${ }^{12}$ Price et al. demonstrated that the rate of re-operation was greatest in the first three years after initial surgery. ${ }^{8,13}$ So far, little is known about specific risk factors for each individual which predispose them to recurrence of POP. ${ }^{13}$ However, recent meta-analyses have shown that surgical procedures can improve quality of life of women with POP, and appropriate choice of surgical technique was found to be among the most important factors on which success of surgery depends. Evidence about which surgical method is the most appropriate for each patient is still lacking. In addition, it is difficult to identify patients with the same degree of POP due to the lack of a universal definition of POP to perform uniform surgery for all of them. ${ }^{12}$ Nevertheless, we tried several surgical techniques to improve our patient's condition; however the proper surgical technique suitable for her could not be found and reoperations were needed. In general, in our hospital recurrences after sacropexy are $4.5 \%$, and after total colpocleisis are less than $1 \%$. To date vaginal hysterectomy is still standard practice, even though descent of the uterus is a result, not a cause of, prolapse. So far, there are some data, which show that the history of POP at the time of hysterectomy has been established as a strong and independent factor for POP recurrence. ${ }^{12}$ In addition, subtotal abdominal hysterectomy does not prevent development of POP compared to total hysterectomy. ${ }^{14}$ Conversely, vaginal apical support procedure is possible to reduce long-term risks for its recurrence. ${ }^{15}$ The success rate of apical fixation procedures is 82$92 \%$. Increasingly, women wish to avoid hysterectomy, because of delay in childbearing and the belief that the uterus is important for sexual satisfaction. To resolve POP and preserve fertility, a sacrospinous uterine fixation is possible. The obliterative procedures such as partial and total colpectomy are reserved for women with stage IIIIV POP who no longer want to preserve coital function, and have a cure rate of 90$97 \%$. In comparison, sacrocolpopexy with a polypropylene mesh has a statistically higher success rate (up to $91 \%$ ) and less postoperative dyspareunia. ${ }^{1}$ However, mesh repairs are also linked with high rates of surgical complications and postoperative adverse effects, and vaginal mesh is banned

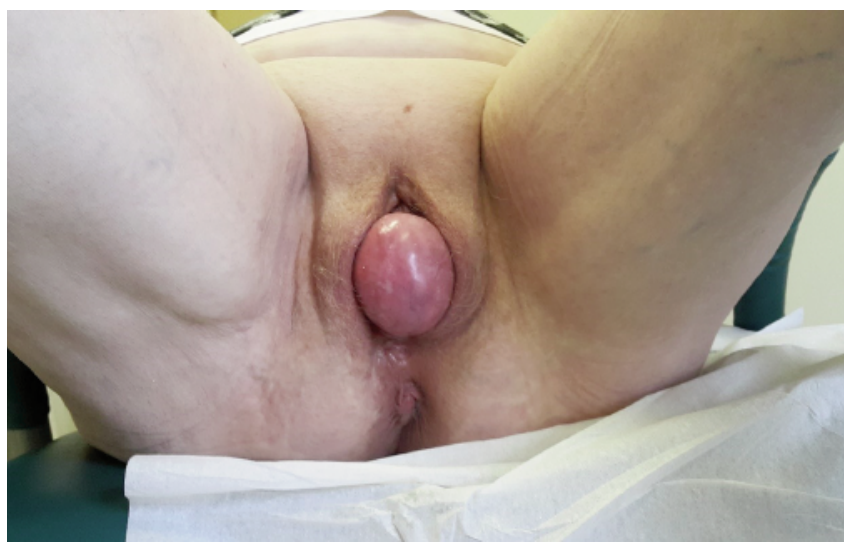

Figure 1. The patient with Stage 3 POP with leading posterior wall.

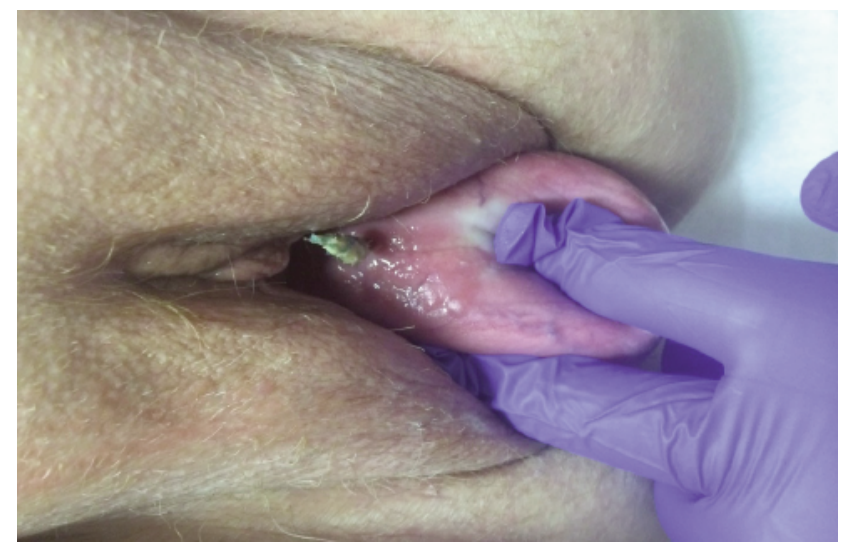

Figure 2. On the examination the posterior mesh that is not fixed to the sacrum anymore and it is eroding trough the mucosa. 
in some countries. ${ }^{16}$

The early postoperative period is fundamental for proper wound healing after pelvic surgery. An increase in abdominal pressure, which may occur at the mobilization, coughing, vomiting and straining at bowel movements, can all threaten normal wound healing. This may influence the success of the surgical procedure and can lead to revision surgeries. ${ }^{12}$ To reduce this risk, some recommend use of vaginal support devices for at least four weeks after surgery. ${ }^{12}$ In our institution, we do not regularly prescribe post-operative use of vaginal support devices, however our patient was always given precise instructions at discharge about how to deal with early and late postoperative periods, such as discouragement of greater physical exertion and heavy lifting, taking care of regular bowel movements and dissuasion of sexual intercourse in the early postoperative period.

Nowadays the influence of genetics on occurrence of POP is emphasized. Vetuschi and coworkers have confirmed a significant influence of collagen imbalance on occurrence of POP. ${ }^{17}$ They have shown that muscularis propria of the vaginal wall in POP patients has more type III collagen than type I, which consists of much thinner fibers, less elastic fibers, and smooth muscle cells were found to be more disorganized. This all results in alternations of architecture and function of muscularis propria. ${ }^{17}$

Presentation of this case clearly demonstrates dramatic recurrences of POP and how difficult its treatment and prevention can be. Even though such extreme cases are infrequent, we wanted to draw attention to them with this case. We need to emphasize that to date the literature of recurrent prolapse does not exist. Our paper is the first contribution, which in detail described the problem of recurrent POP. To understand why relapse occurs and why in our patient, despite so many different surgeries, relapse always occurred, we would need clinical studies focusing on identifying risk factors for each patient. Only individual treatment and individual selection of therapy could avoid recurrence of POP. To avoid development and reoccurrences of POP it is necessary to identify high-risk populations and focus on modification of risk factors. It is important to develop new techniques for the patients with recurrent prolapse, to spare patients future re-admissions for future surgeries.

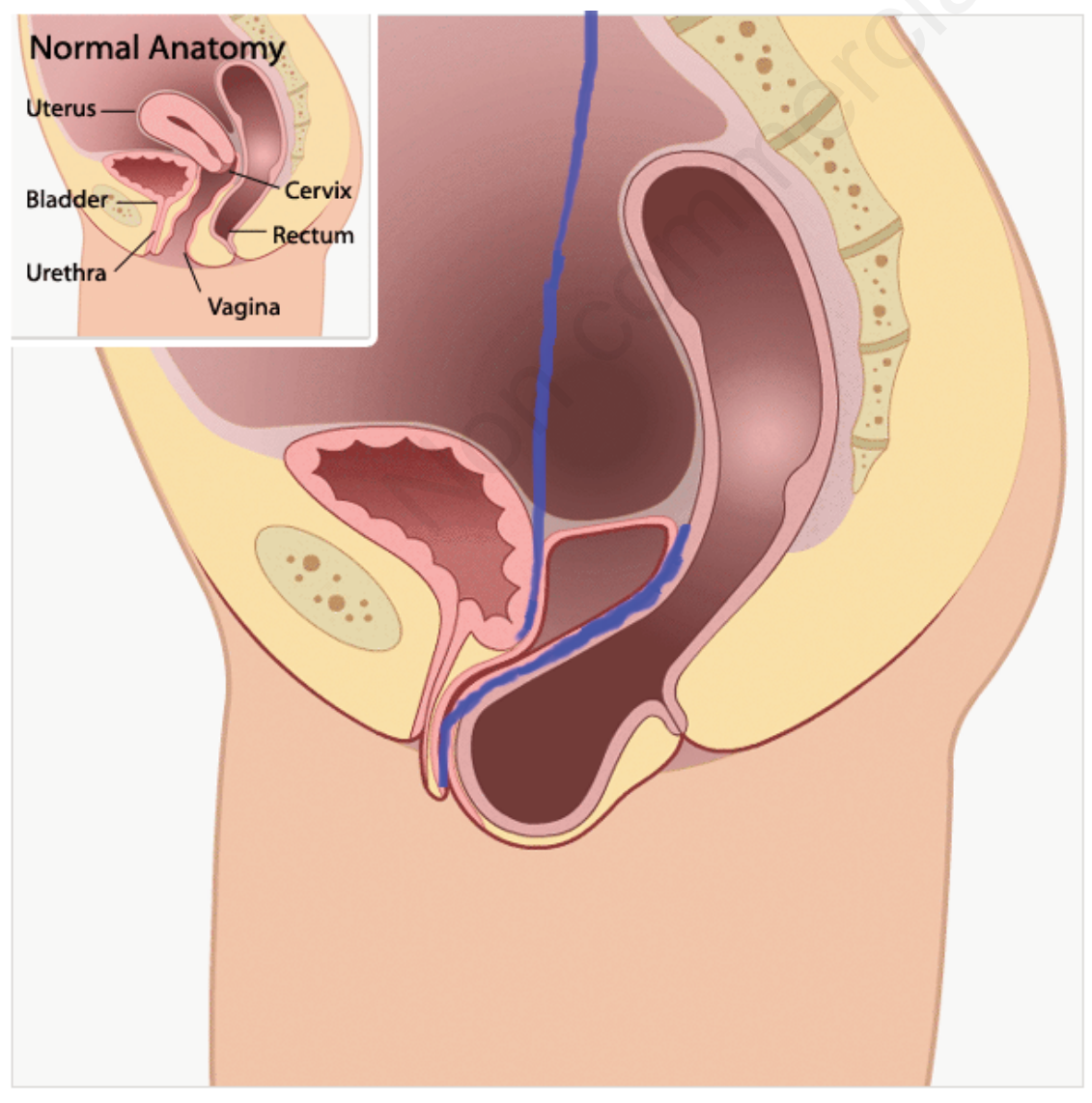

Figure 3. The loose posterior mesh is seen on the picture. The anterior mesh is still in the place. Both meshes are presented in blue color.

\section{Conclusions}

POP is one of the leading pathologies, which can have extremely negative impact on quality of life of individual women. Despite the fact that we expect marked increase in the incidence of POP over the next 30 years, little is known about modifiable and non-modifiable risk factors and appropriate treatment options for each individual. Therefore, we recommend the implementation of future researches with uniform definition of POP, which would be more explicit and easier to compare with one another. In addition, randomized studies about recurrence of POP are needed, since there is no data on this topic in the literature yet.

\section{References}

1. Barber MD, Maher C. Epidemiology and outcome assessment of pelvic organ prolapse. Int Urogynecol J 2013;24:1783-90.

2. De Vita D, Giordano S. Two successful natural pregnancies in a patient with severe uterine prolapse: a case report. J Med Case Rep 2011;14;5:459.

3. Bump R, Norton PA. Epidemiology and natural history of pelvic floor dysfunction. Obstet Gynecol Clin North Am 1998;25:723-46.

4. Diez-Itza I, Aizpitarte I, Becerro A. Risk factors for the recurrence of pelvic organ prolapse after vaginal surgery: a review at 5 years after surgery. Int Urogynecol J Pelvic Floor Dysfunct 2007;18:1317-24.

5. Gyhagen M, Bullarbo M, Nielsen TF, Milsom I. Prevalence and risk factors for pelvic organ prolapse 20 years after childbirth: a national cohort study in singleton primiparae after vaginal or caesarean delivery. BJOG 2013;120:152-60.

6. Whiteside J, Weber A, Meyn LA, Walters MD. Risk factors for prolapse recurrence after vaginal repair. Am J Obstet Gynecol 2004;191:1533-8.

7. Tegerstedt G, Hammarström M. Operation for pelvic organ prolapse: a follow-up study. Acta Obstet Gynecol Scand 2004;83:758-63.

8. Price N, Slack A, Jwarah E, Jackson S. The incidence of reoperation for surgically treated pelvic organ prolapse: an 11-year experience. Menopause Int 2008;14:145-8.

9. Cheung RY, Lee JH, Lee LL, Chung TK, Chan SS. Vaginal pessary in women with symptomatic pelvic organ 
prolapse: a randomized controlled trial. Obstet Gynecol 2016 [Epub ahead of print].

10. Brubaker L, Maher C, Jacquetin B, et al. Surgery for pelvic organ prolapse. Female Pelvic Med Reconstr Surg 2010;16:9-19.

11. Schull BL, Bachofen C, Coates KW, Kuehl TJ.A transvaginal approach to repair of apical and other associated sites of pelvic organ prolapse with uterosacral ligaments. Am J Obstet Gynecol 2000;183:1365-74.

12. Salvatore S, Siesto G, Serati M. Risk factors for recurrence of genital prolapse. Curr Opin Obstet Gynecol
2010;22:420-4.

13. Costa J, Towobola B, McDowel C, Ashe R. Recurrent pelvic organ prolapse (POP) following traditional vaginal hysterectomy with or without colporrhaphy in an Irish population. Ulster Med J 2014;83:16-21.

14. Persson P, Brynhildsen J, Kjølhede P. Pelvic organ prolapse after subtotal and total hysterectomy: a long-term followup of an open randomised controlled multicentre study.

BJOG 2013;120:1556-65.

15. Eilber KS, Alperin M, Khan A, et al. Outcomes of vaginal prolapse surgery among female Medicare beneficiaries: the role of apical support. Obstet Gynecol 2013;122:981-7.

16. Jacklin P, Duckett J. A decision-analytic Markov model to compare the cost-utility of anterior repair augmented with synthetic mesh compared with nonmesh repair in women with surgically treated prolapse. BJOG 2013;120:21723.

17. Vetuschi A, D'Alfonso A, Sferra R, et al. Changes in muscularis propria of anterior vaginal wall in women with pelvic organ prolapse. Eur J Histochem 2016;60:2604 